\title{
Foreign Body in Jugal Mucosa
}

\author{
Thiago Luís Infanger Serrano ${ }^{1}$ Henrique Furlan Pauna ${ }^{1}$ Igor Moreira Hazboun ${ }^{1}$ Ana Cristina Dal Rio ${ }^{2}$ \\ Maria Elvira Pizzigatti Correa ${ }^{2}$ Ester Maria Danielli Nicola ${ }^{1}$
}

${ }^{1}$ Department of Otolaryngology, Head and Neck Surgery, Universidade Estadual de Campinas (UNICAMP), Campinas, São Paulo, Brazil

${ }^{2}$ Department of Otorhinolaringology and Multidisciplinary Laser Unit, UNICAMP, Campinas, São Paulo, Brazil

Int Arch Otorhinolaryngol 2015;19:364-366.
Address for correspondence Thiago Luís Infanger Serrano, ENT Resident, Department of ENT, UNICAMP, Av. Albert Einstein, Cidade Universitária "Zeferino Vaz" Barão Geraldo, Campinas, SP Campinas 13081-970, Brazil (e-mail: thiagoserrano@uol.com.br).

\section{Abstract \\ Keywords \\ - foreign body reaction \\ - granulomatosis \\ - orofacial \\ - differential diagnoses \\ - esthetic surgery}

Introduction Foreign body in the oral cavity may be asymptomatic for long time and only sometimes it can lead to a typical granulomatous foreign body reaction. Some patients may complain of oral pain and present signs of inflammation with purulent discharge. A granuloma is a distinct, compact microscopic structure composed of epithelioid-shaped macrophages typically surrounded by a rim of lymphocytes and filled with fibroblasts and collagen. Nowadays, the increase of cosmetic invasive procedures such as injection of prosthetic materials in lips and cheeks may lead to unusual forms of inflammatory granulomas.

Objectives Describe an unusual presentation of a foreign body reaction in the buccal mucosa due to previous injection of cosmetic agent.

Resumed Report A 74-year-old woman was referred to the Department of Otorhinolaryngology, Head and Neck Surgery to investigate the presence of multiple painless, bilateral nodules in the buccal mucosa, with progressive growth observed during the previous 2 months. The histologic results showed a foreign body inflammatory reaction. Conclusion Oral granulomatosis lesions represent a challenging diagnosis for clinicians and a biopsy may be necessary. Patients may feel ashamed to report previous aesthetic procedures, and the clinicians must have a proactive approach.

\section{Introduction}

The demand for aesthetic surgery has been increasing, and some materials, such as silicone, polymethyl methacrylate, collagen, and hyaluronic acid, are commonly used in plastic surgery. ${ }^{1,2}$ When used in soft tissues, these materials can stay latent for a long time. Sometimes they may cause chronic inflammatory reaction. ${ }^{3}$

Granulomatous inflammation of the oral soft and hard tissues is an uncommon occurence. ${ }^{4}$ Clinically, it can be presented with a local inflammatory reaction that can be associated with purulent discharge. ${ }^{1}$ Local pain can vary among patients. The incisional biopsy is the exam of choice in the diagnosing process. ${ }^{4}$

received

November 21, 2014

accepted

January 26, 2015

published online

March 13, 2015
DOI http://dx.doi.org/

10.1055/s-0035-1547522. ISSN 1809-9777.
The objective of this case report is to highlight the clinical presentation of a foreign body reaction in the oral cavity as a late effect of facial plastic procedure.

Cases that present as solid facial swellings should be differentiated especially from tuberculosis, facial erysipelas, South American blastomycosis, angioedema, orofacial granulomatosis, Crohn disease, and sarcoidosis. These diseases are detailed in the Discussion, along with the recent literature about the subject.

\section{Case Report}

A 74-year-old woman was referred to the otorhinolaryngology department of a tertiary hospital for oral examination. At the

Copyright $\odot 2015$ by Thieme Publicações License terms Ltda, Rio de Janeiro, Brazil 
patient's first appointment, she complained of the presence of painless hard nodules in the buccal mucosa, bilaterally, with 2 months of evolution. According with patient's report, the hard nodules were more evident during the morning, and they were always accompanied by local edema in the malar region. The nodules diminished in size during the day. Palpation of the oral tissues revealed several small nodules $(1 \mathrm{~cm}$ diameter each) bilaterally. The nodules were not regularly distributed along the malar region and were spread out in the muscle area. During anamneses, the patient denied any plastic surgery in the past. With the probable diagnosis of deposition disease, a local incisional biopsy was performed (-Fig. 1). The biopsy results showed a granulomatous reaction, caused probably by an exogenous foreign body (-Fig. 2-3).

To determine the patient's past medical history, a slide review was requested, which confirmed the previous histopathologic results. The confirmation of the previous facial filling was stated by the patient's caregiver, who said that the patient had facial plastic surgery 20 years before in a private clinic, which explained the lack of this information on the patient's medical records in our hospital.

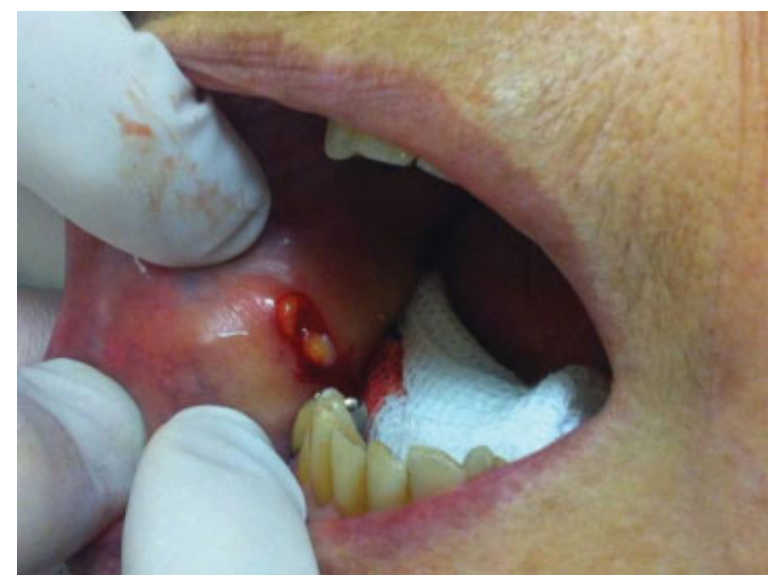

Fig. 1 Biopsy showing the yellowish coloration and hard consistency from right buccal mucosa.

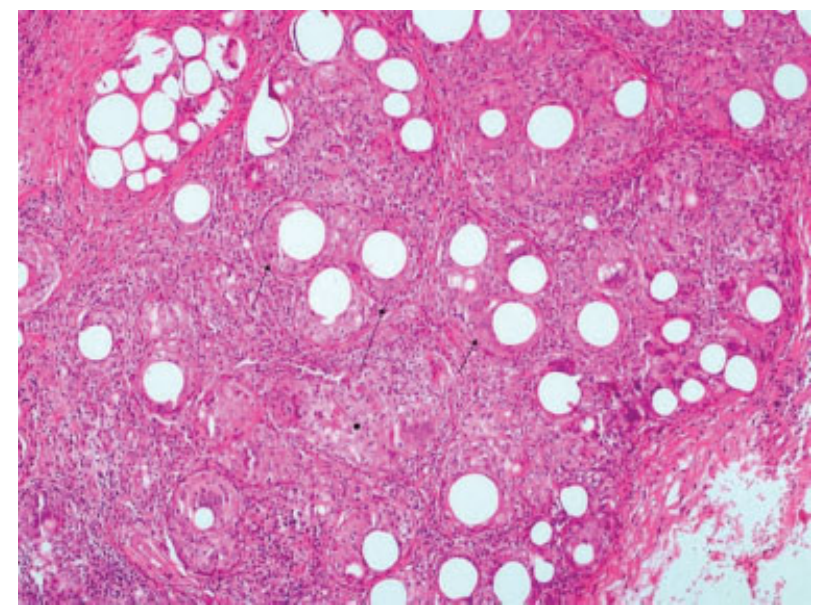

Fig. 2 Histopathologic view (hematoxylin and eosin $10 \times$ ) showing epithelioid-shaped macrophages surrounded by a rim of lymphocytes (full arrows), filled with fibroblasts and collagen (arrow with ball head).

\section{Discussion}

Foreign body inflammatory reaction can appear from a few months to several years after a surgical procedure. ${ }^{5}$ It may result from local trauma or it may be iatrogenic. ${ }^{6}$ Because of the increasing number of aesthetic procedures and the use of different biomaterials, foreign body inflammatory reaction may become more frequent. These reactions show a female predilection, probably reflecting the fact that women seek cosmetic care more often than men. ${ }^{2}$

A granuloma is a distinct, compact microscopic structure composed of epithelioid-shaped macrophages typically surrounded by a rim of lymphocytes and filled with fibroblasts and collagen. Multinucleated giant cells are also present and form from coalescing epithelioid macrophages. ${ }^{4}$

Foreign materials are composed of particles that are usually too large to be phagocytosed and are the most common source of granulomatous inflammation in the oral cavity. ${ }^{4}$ They do not evoke an immune response because they are typically inert, but there is a macrophage recruitment to eliminate the material. The identification of the foreign material is not easy and sometimes it is necessary to use polarized light to visualize them. ${ }^{4}$

Because of the relatively nonspecific clinical findings associated with a variety of granulomatous diseases, a microscopic diagnosis of granulomatous inflammation often presents a diagnostic dilemma. ${ }^{4}$ The differential diagnosis may include a broad range of conditions. Cases that present as solid facial swellings should be differentiated especially from tuberculosis, facial erysipelas, South American blastomycosis, angioedema, orofacial granulomatosis, Crohn disease, and sarcoidosis. A clinical presentation of multiple nodules should be distinguished from Heck disease, neurofibromatosis, amyloidosis, multiple endocrine neoplasia type $2 \mathrm{~b}$ syndrome, and lipoid proteinosis. ${ }^{2,4,7}$

Orofacial granulomatosis is a nonspecific, descriptive term encompassing a variety of conditions that exhibit similar

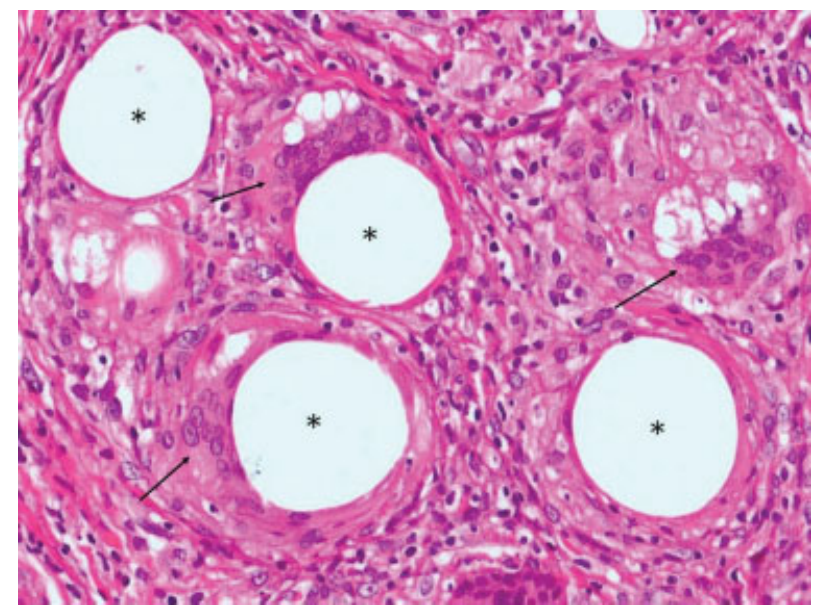

Fig. 3 Histopathologic view (hematoxylin and eosin $40 \times$ ): multinucleated giant cells with haphazardly/peripherally nuclei shape (arrows), associated with areas without substance, indicating foreign body particles $\left({ }^{*}\right)$. 
clinical and microscopic features. It is used as a clinical diagnosis of exclusion, and its exact cause remains unknown. The most consistent finding is a persistent, painless swelling of the orofacial tissues, with vertical fissure of the lips the most frequent involvement. ${ }^{4}$

The early diagnostic and detection of foreign bodies are based at the anamnesis and physical examination. ${ }^{5}$ In longterm follow-up, patients may present with oral pain and signs of inflammation with purulent discharge, granulomas, and migration of material. ${ }^{1,2}$ Migration of foreign particles is a rarely reported phenomenon that could be explained by three mechanisms: hematogenous spread of particles, lymphatic injection, and phagocytosis of the particles by macrophages that travel through the lymphatic system to the local lymph nodes. ${ }^{7}$ Reports of asymptomatic foreign bodies affecting the oral cavity are rarely reported in the literature. ${ }^{1,2}$

In the case reported here, the patient presented nodular lesions without pain or discharge and denied previous plastic procedures at the first visit. The oral examination was inconclusive. Imaging exams are helpful, with routine radiography being the preferred imaging modality for the initial workup. However, several types of soft tissue foreign bodies are not radiopaque and therefore remain undetected. Sonography is playing an increasing role in the diagnostic process and for accurate localization of all types of soft tissue foreign bodies, minimizing surgical exploration or, alternatively, guiding percutaneous removal of foreign body. ${ }^{8}$

Surgical excision has been reported as the preferred option for well-circumscribed nodular lesions. ${ }^{6,7}$ Surgery for widespread lesions remains controversial. Some authors argue that complete eradication of the granulomatous tissue is highly improbable and that surgery may lead to scarring and fistulas. In the other hand, González-García et al argue that surgery combined with corticoids is the preferred option for these cases. ${ }^{7}$

\section{Final Comments}

Foreign body reaction as a late effect after plastic surgery may be very uncommon. However, when it occurs, biopsy is the standard diagnostic procedure, along with a good anamneses process and past medical history.

\section{References}

1 Puliyel D, Balouch A, Ram S, Sedghizadeh PP. Foreign body in the oral cavity mimicking a benign connective tissue tumor. Case Rep Dent 2013;2013:369510

2 Jham BC, Nikitakis NG, Scheper MA, Papadimitriou JC, Levy BA, Rivera H. Granulomatous foreign-body reaction involving oral and perioral tissues after injection of biomaterials: a series of 7 cases and review of the literature. J Oral Maxillofac Surg 2009;67(2): 280-285

3 Manthey DE, Storrow AB, Milbourn JM, Wagner BJ. Ultrasound versus radiography in the detection of softtissue foreign bodies. Ann Emerg Med 1996;28(1):7-9

4 Alawi F. Granulomatous diseases of the oral tissues: differential diagnosis and update. Dent Clin North Am 2005;49(1):203-221, x

5 Silveira VAS, de Carmo ED, Colombo CED, Cavalcante ASR, Carvalho YR. Intraosseous foreign-body granuloma in the mandible subsequent to a 20-year-old work-related accident. Med Oral Patol Oral Cir Bucal 2008;13(10):E657-E660

6 Aregbesola SB, Ugboko VI. Unusual foreign bodies in the orofacial soft tissue spaces: a report of three cases. Niger J Clin Pract 2013; 16(3):381-385

7 González-García R, Rodríguez-Campo FJ, Román-Romero L, et al. Migration of aluminum silicate from the oral cavity to the submandibular region, with foreign body granuloma formation: report of a case. Oral Surg Oral Med Oral Pathol Oral Radiol Endod 2007;104:e45-e49

8 Horton LK, Jacobson JA, Powell A, Fessell DP, Hayes CW. Sonography and radiography of soft-tissue foreign bodies. AJR Am J Roentgenol 2001;176(5):1155-1159 